\title{
Coordinate Detection for Automatic Guided Vehicle Using Indoor Ceiling LED Lights
}

\author{
Shiyuan Yang ${ }^{*}$, Kenta Kagemoto, Kosuke Onishi \\ Kyushu Institute of Technology, 1-1 Sensui-cho, Tobata-ku, Kitakyushu, Fukuoka, 804-8550, Japan \\ *Corresponding Author: yang@elcs.kyutech.ac.jp
}

\begin{abstract}
Automatic guided vehicles (AGVs) are widely used in various manufacturing factory such as automobile fabrication factory. The popular guiding method is magnetic induction method, but it needs a lot of effort and time when the travel route is changed. In this study, we proposed a new method to detect the coordinate of an AGV for its auto guiding. The indoor ceiling light emitting diode (LED) lights are utilized as lighting marker, which are modulated with different frequencies. A photodiode is set on the AGV and receive the LED light to determine the coordinates of the AGV. Experimental results showed this new method can achieve enough accuracy under $50 \mathrm{~cm}$ for AGV coordinate detection.
\end{abstract}

Keywords: Automatic Guided Vehicle (AGV), Light Emitting Diode (LED), Photodiode, Coordinate detection.

\section{Introduction}

Self-location detection is widely used for automatic running device such as autonomous cars, robots, automatic guided vehicles (AGVs), etc. A typical Self-location detection device is global positioning system (GPS), but it cannot be used indoor. In general, AGVs used in manufacture factory usually use magnetic induction method for automatic running. It takes effort and time when the travel route is changed. Other guiding systems such as gyro position detection and laser position detection had been suggested. The gyro position detection has large accumulative error and need marker for position correction. The laser position detection need accurate reflectors that cost effort for setting. Camera systems are often used for position detection, but it requires landmark data and the map data previously ${ }^{(1-3)}$. In addition, a camera system is expensive for introducing. In a previous study, the authors have suggested a method for position detection of moving objects using a two dimensional position sensitive detector (PSD) and the ceiling LED Lights ${ }^{(4)}$. This method can detect self-location accurately and can change the travel route easily.

In this study, we propose a new method using a photodiode and multiple ceiling LED lights sine waved with different frequency. Instead of a PSD by a photodiode, the position detection can be constructed cheaply and the position error is small enough for moving object.

\section{Construction of coordinate detection using a photodiode and multiple LED lights}

The construction of coordinate detection using a photodiode and multiple LED lights is shown in Fig. 1. The LED lights are set on the ceiling plane with same distances and sine waved with different frequency. The photodiode is set on a vehicle and receive the lights from LEDs. After frequency analysis with Fourier transform, the light intensity of different LED can be obtained.

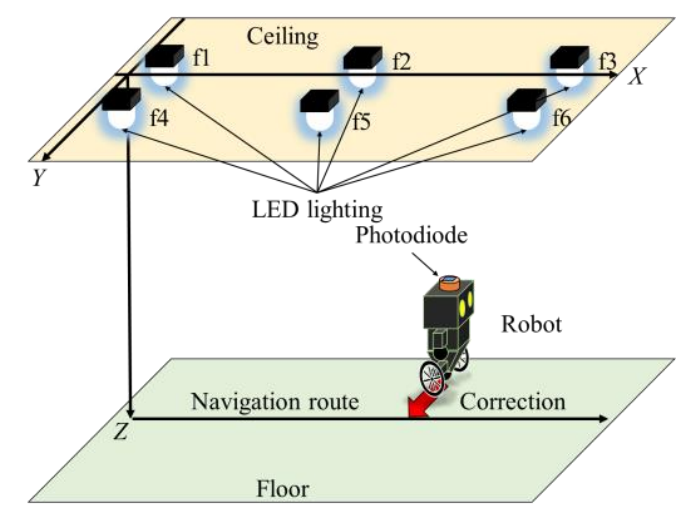

Fig. 1 Construction of coordinate detection using a photodiode and multiple LED lights. 
For a general LED, the light amplitude spectrum distribution can be expressed as

$$
I(R)=I_{\max }\left\{\cos \left(\tan ^{-1} \frac{R}{Z}\right)\right\}^{4}
$$

where $R$ is the horizontal radius from the LED light center, $Z$ is the vertical distance, $I_{\max }$ is the LED source amplitude spectrum. The theoretical distribution of an LED light is shown in Fig. 2. Its experimental distribution is shown in Fig.3. From these results, we can see that the real measurement is mostly satisfying Eq. (1). By changing Eq. (1), we have

$$
R=Z \tan \left\{\cos ^{-1}\left(\sqrt[4]{\frac{I(R)}{I_{\max }}}\right)\right\}
$$

When the LED light amplitude spectrum is measured by the photodiode, the horizontal radius from the LED light center $R$ is obtained according to Eq. (2). The position with $R$ is on a circle as shown in Fig. 4 .

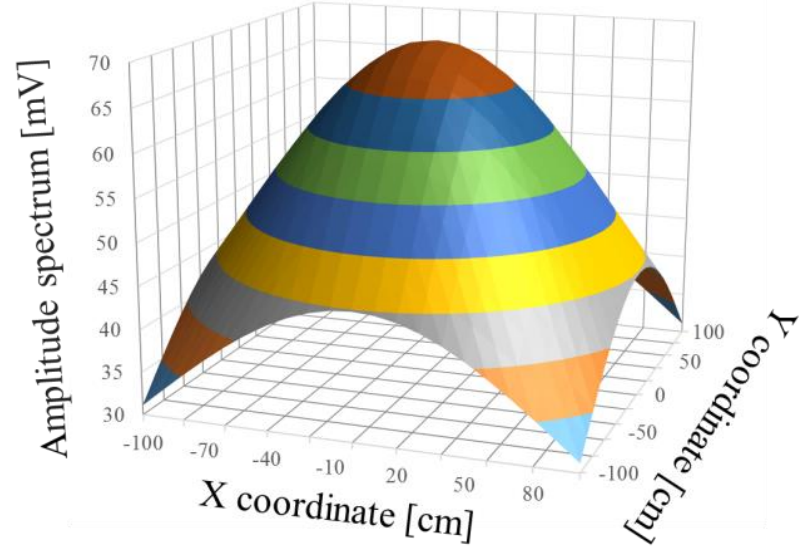

Fig. 2 Theoretical distribution of an LED light.

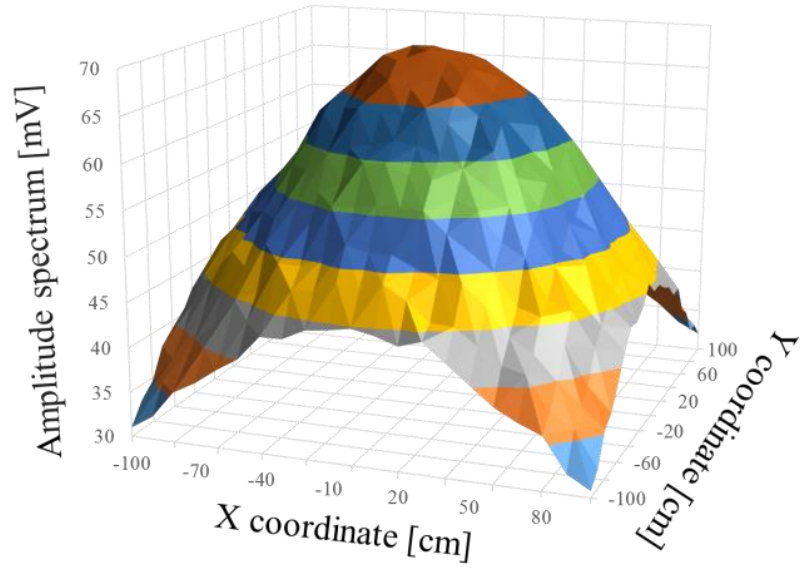

Fig. 3 Experimental distribution of an LED light.
When two LED lights are used, the position is at the intersection point of two circle as shown in Fig.5. And the position is determined when more than three LED lights are used as shown in Fig. 6. And the accuracy can be improved by increasing the number of LED lights.

According to Fig. 6, supposing the LEDs are set at coordinate $(\mathrm{a}, \mathrm{b}),(\mathrm{c}, \mathrm{d})$ and $(\mathrm{e}, \mathrm{f})$, we can determine the coordinate of the photodiode by the following equation

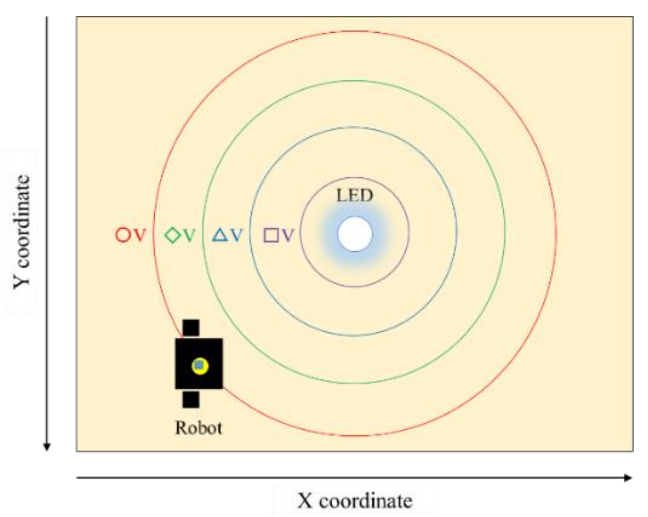

Fig. 4 Position detection using one LED light.

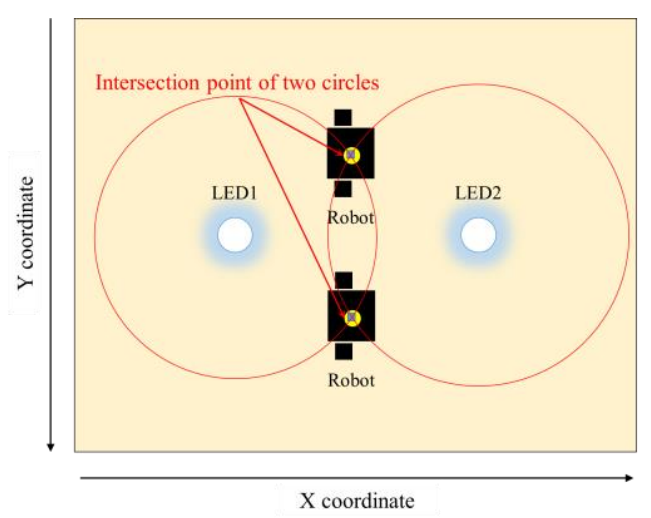

Fig. 5 Position detection using two LED lights.

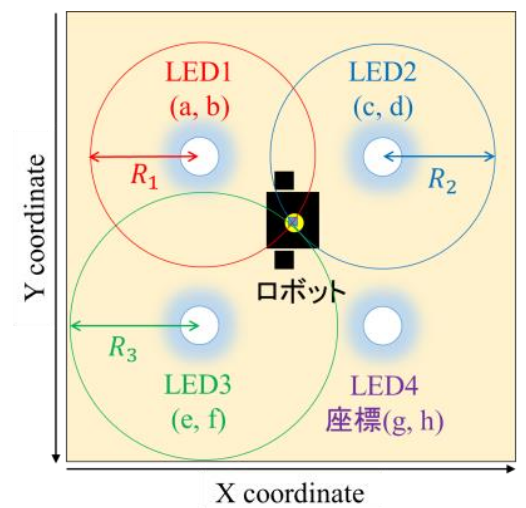

Fig. 6 Position detection using three LED lights. 


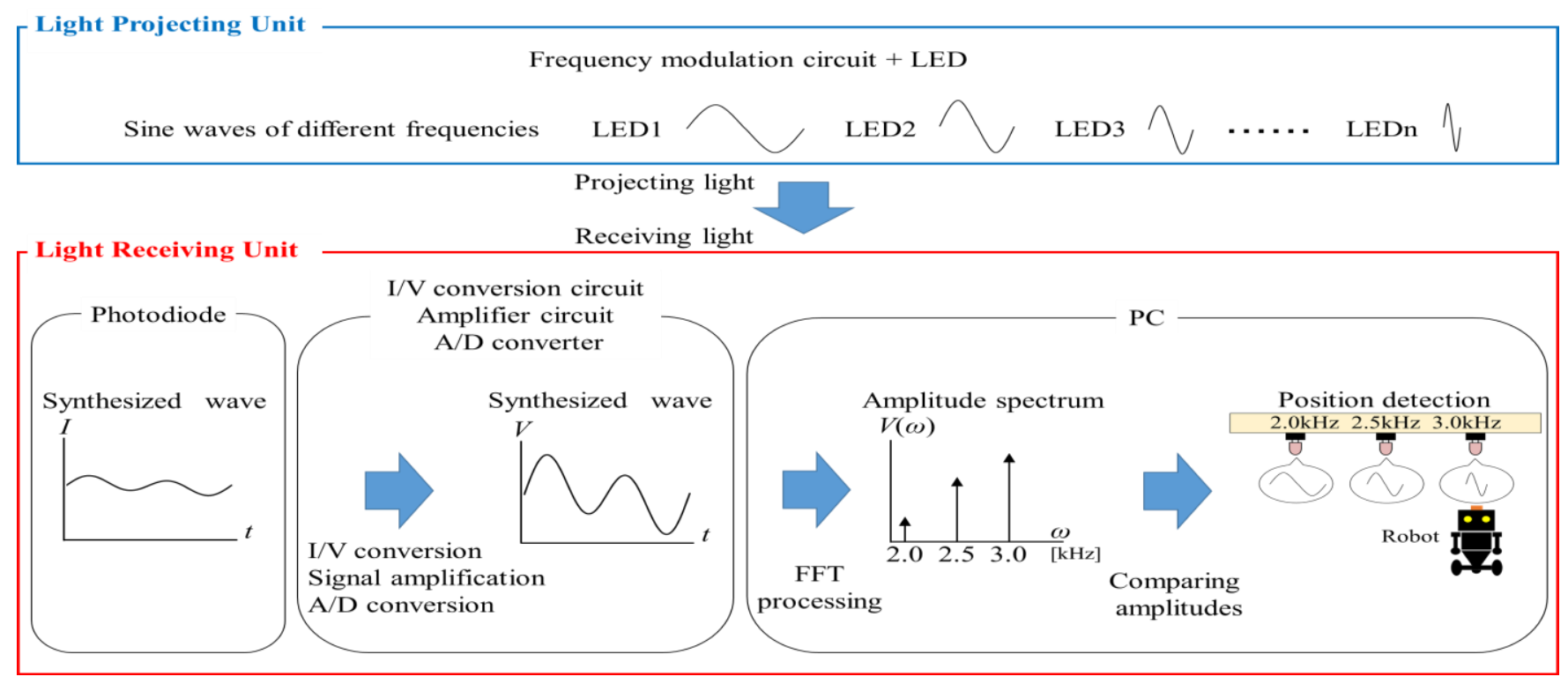

Fig. 7 Signal processing flow for coordinate detection.

$X=$
$\frac{\left\{\left(R_{1}{ }^{2}-R_{2}{ }^{2}\right)-\left(a^{2}+b^{2}-c^{2}-d^{2}\right)\right\}(d-f)+\left\{\left(R_{3}{ }^{2}-R_{2}{ }^{2}\right)+\left(c^{2}+d^{2}-e^{2}-f^{2}\right)\right\}(b-d)}{2\{(-a+c)(d-f)+(b-d)(c-e)\}}$
$Y=$
$\frac{\left\{\left(R_{1}{ }^{2}-R_{2}{ }^{2}\right)-\left(a^{2}+b^{2}-c^{2}-d^{2}\right)\right\}(c-e)+\left\{\left(R_{3}{ }^{2}-R_{2}{ }^{2}\right)+\left(c^{2}+d^{2}-e^{2}-f^{2}\right)\right\}(a-c)}{2\{(-b+d)(c-e)+(a-c)(d-f)\}}$

$\frac{\left\{\left(R_{1}{ }^{2}-R_{2}{ }^{2}\right)-\left(a^{2}+b^{2}-c^{2}-d^{2}\right)\right\}(c-e)+\left\{\left(R_{3}{ }^{2}-R_{2}{ }^{2}\right)+\left(c^{2}+d^{2}-e^{2}-f^{2}\right)\right\}(a-c)}{2\{(-b+d)(c-e)+(a-c)(d-f)\}}$

where

$$
R_{\mathrm{i}}=Z \tan \left\{\cos ^{-1}\left(\sqrt[4]{\frac{I\left(R_{i}\right)}{I_{\max }}}\right)\right\} \quad(i=1,2,3,4)
$$

In addition, other three LEDs combinations can be also used to determine the position coordinate and the error can be decreased by averaging the values of four combinations.

Coordinate detection can be achieved using the above analysis and the construction of Fig.1. The signal processing flow is shown in Fig. 7. At first, the ceiling LED are sine waved with different frequency and illuminate vertically. The coordinate of each LED is measured previously and can be known from its frequency information. The photodiode is set on the vehicle horizontally and receive the synthesized light wave. By making Fourier transform of this synthesized signal, one can get the amplitude spectrum of each frequency. Using the strongest three amplitude spectra that corresponding to the nearest three LEDs, the coordinate of the vehicle can be calculated using Eqs. (3) and (4).

Figure 8 shows an example of amplitude spectra measurement and coordinate calculation. More than the

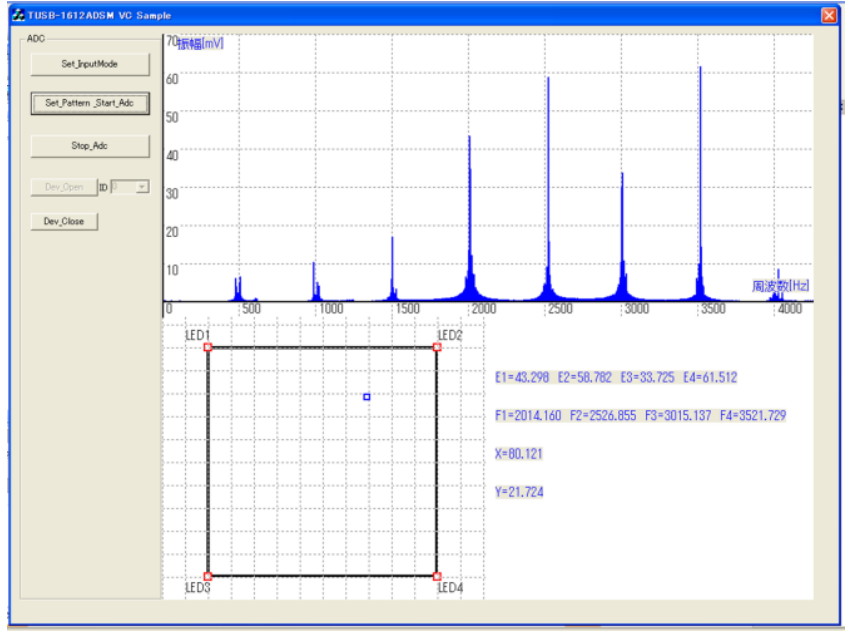

Fig. 8. Example of spectra measurement and coordinate calculation

strongest three amplitude spectra could be used but it leads to a large error when other spectra are weak.

\section{Experiments}

Coordinate detection was performed in the square surrounded by four LEDs. The distances between LEDs are one meter and the distance between the photodiode and ceiling plane is two meter. The four LEDs are sine waved 


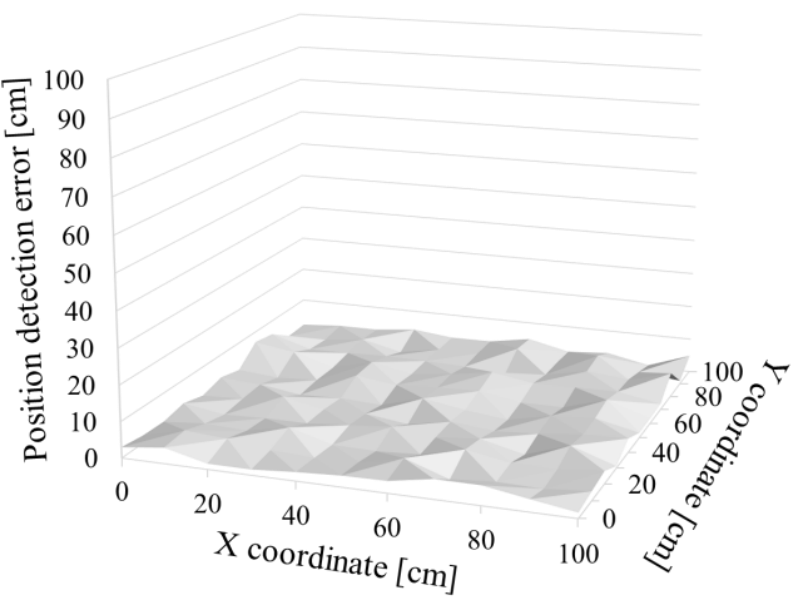

Fig. 9 Error distribution of distance between the measured position and the real one.

with frequencies of $2.0 \mathrm{kHz}, 2.5 \mathrm{kHz}, 3.0 \mathrm{kHz}$ and $3.5 \mathrm{kHz}$, respectively. The position of the diode is set at coordinate from $(0,0)$ to $(100,100)$ with intervals of 10 centimeters. Figure 9 shows the error distribution of distance between the measured position and the real one. The maximum error is about 5 centimeters so it is possible for AGV coordinate detection. In addition, the processing time is about 50 milliseconds so it is enough for an AGV movement under the speed of $1 \mathrm{~m} / \mathrm{s}$ indoor.

\section{Conclusions}

A new approach is suggested for the coordinate measurement of $\mathrm{AGV}$ using a photodiode and multiple ceiling LED lights sine waved. It has the merit of low cost construction and easy change for travel route of an AGV. Experimental results showed that the coordinate measurement is enough for the guide of an AGV. This approach overcomes the difficulty of coordinate measurement indoor such as using a GPS system and can be applied in the applications of conventional AGVs. In addition, it can be also used as a calibration reference for other coordinate measurement method such as the gyro position detection method. As a future study, we will use multiple photodiodes to overcome the difficulty of keeping the photodiode horizontally.

\section{Acknowledgment}

This study is partially supported by the 2016 research grant of Kitakyushu foundation for the advancement of industry science and technology.

\section{References}

(1) Oe, Motoko, Tomokazu Sato, and Naokazu Yokoya: "Estimating camera position and posture by using feature landmark database", Scandinavian Conference on Image Analysis. Springer Berlin Heidelberg, pp. 171181,2005

(2) Nor Aida Mahiddin, Elissa Nadia Madi, Siti Dhalila, Engku Fadzli Hasan, Suhailan Safie and Noaizan Safie: "User Position Detection In An Indoor Environment", International Journal of Multimedia and Ubiquitous Engineering, Vol.8, No.5, pp.303-312, 2013

(3) Yoshino Masaki, Shinichiro Haruyama, Masao Nakagawa: "High-accuracy positioning system using visible LED lights and image sensor", 2008 IEEE Radio and Wireless Symposium. IEEE, pp.439-442, 2008

(4) Kosuke Onishi, Daisuke Tanaka, Shiyuan Yang: "3D Position Detection Using a 2D PSD and a Marker Composed of Light Sources", Journal of the Institute of Industrial Applications Engineers, Vol.4, No2, pp. 8693, 2016 